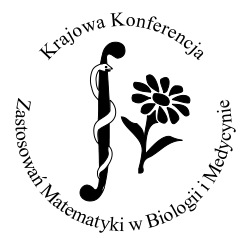

Jastrzębia Góra, $16^{\text {th }}-20^{\text {th }}$ September 2013

\title{
ARITHMETICAL ANALYSIS OF BIOMOLECULAR FINITE AUTOMATON
}

\author{
Tadeusz Krasiński ${ }^{1}$, Sebastian Sakowski ${ }^{1}$, Jacek Waldmajer ${ }^{2}$, Tomasz Popławski ${ }^{3}$ \\ ${ }^{1}$ Faculty of Mathematics and Computer Science, University of Łódź \\ Banacha 22, 90-238 Łódź, Poland \\ ${ }^{2}$ Group of Logic, Language and Information, University of Opole \\ Katowicka 89, 45-061 Opole, Poland \\ ${ }^{3}$ Department of Molecular Genetics, University of Lódź \\ Pomorska 141/143, 90-236 Lódź, Poland,

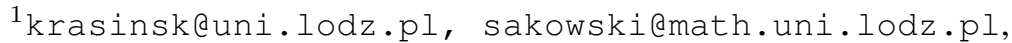 \\ 2 jwaldmajer@uni.opole.pl, ${ }^{3}$ tplas@iol.uni.lodz.pl
}

\begin{abstract}
We present the result of the article [1], in which a theoretical analysis of extension of the finite automaton built on DNA (introduced by the Shapiro team) to an arbitrary number of states and symbols is given. The Shapiro automaton action is based on cyclic cutting and ligating of DNA molecules. Its distinctive features included the fact that it was programmable (it means we may choose arbitrarily transitions of any two-state automaton), as well as autonomous (the activity of the automaton does not require man's intervention during calculations). However the Shapiro automaton was only 2-state 2-symbol finite automaton. The first two authors proposed in [2] use a new idea of several restriction enzymes instead of one to extend numbers of states in Shapiro automaton. In the article [1] we present conditions for the existence of such extensions in terms of ingredients used in the implementation. We give arithmetical conditions for numbers of states, symbols, length of codes, the number of used restriction enzymes and length of leaving sticky ends under which it would be possible to construct more powerful biomolecular computers. We also analyze from theoretical point of view the idea of increasing the complexity of this automata by using many restriction enzymes (instead of one) in the same mixture. Another theoretical analysis, precisely computational power of the Shapiro automata (called by the authors the Benenson automata) was given by Soloveichik and Winfree [3].

Our next future work will be concentrated on construction of algorithms and computer software to build elements of DNA finite automaton in the form of DNA strands (satisfying required conditions) for laboratory experiments.
\end{abstract}

\section{ACKNOWLEDGEMENTS}

This project is supported by the National Science Centre of Poland (NCN). Grant number: DEC2011/01/B/NZ2/03022.

\section{REFERENCES}

[1] Krasiński T., Sakowski S., Waldmajer J., and Popławski T.: Arithmetical analysis of biomolecular finite automaton, Fundamenta Informaticae, 2013 (in press).

[2] Krasiński T. and Sakowski S.: Extended Shapiro Finite State Automaton. Foundations of Computing and Decision Science, Foundations of Computing and Decision Science 33 (2008), 241-255.

[3] Soloveichik D. and Winfree E.: The Computational Power of Benenson Automata, Theoretical Computer Science 344 (2005), 279-297. 